\title{
Asociación de síndrome metabólico y nefrolitiasis
}

\author{
Metabolic syndrome and nefrolitiasis association
}

\author{
Mariana Araceli Oseguera-Brizuela*
}

\begin{abstract}
RESUMEN. La prevalencia de cálculos renales se ha duplicado en las últimas tres décadas, es una de las principales causas de morbilidad y tiene alto impacto económico. La fisiopatología de la formación de cálculos es un proceso complejo e incluye diversos componentes como volumen urinario bajo, hipercalciuria, hiperoxaluria, hipocitraturia y anormalidades en el pH urinario, los cuales tienen relación importante con cada uno de los componentes del síndrome metabólico, y todo esto se ve relacionado con los tipos de litos más frecuentes en estos pacientes. Cada uno de los componentes del síndrome metabólico tiene un impacto importante en el metabolismo de los pacientes, provocando cambios que hacen que sean más propensos a desarrollar cálculos renales. Éstos ocasionan alto riesgo de padecer enfermedades como enfermedades cardiovasculares, insuficiencia renal, y padecimientos del tracto urinario, que es el que se ve mayormente afectado por la obstrucción y deterioro epitelial causados por los litos. El tratamiento de nefrolitiasis en pacientes con síndrome metabólico se centra en modificar y revertir todos estos cambios. Este tratamiento se compone de cambios en el estilo de vida y de fármacos que ayudan a la modificación de estos factores que se encuentran alterados.
\end{abstract}

Palabras clave: Síndrome metabólico, litos, riesgo cardiovascular, estrés oxidativo, ácido úrico, hipercalciuria.

ABSTRACT. The prevalence of kidney stones are doubled in the last three decades, is one of the principal causes of morbidity and has a high economic impact. The pathophysiology of stone formation is a complex process that includes many components like low urinary volume, hypercalciuria, hyperoxaluria, hypocitraturia, urinary $\mathrm{pH}$ abnormalities, there are importantly related to the components of the metabolic syndrome, and on the other hand, it is related with the most frequent types of stones in this type of patients. The components of metabolic syndrome has an important impact on the metabolism of patients who suffer this diseases, causing changes that bring more risk to develop kidney stones, these patients have a high risk of suffering from various aggregate diseases, among which are cardiovascular diseases, renal failure, and many diseases of the urinary tract, which is one of the most affected by the obstruction and it have an epithelial risk caused by the lithos. The treatment of nephrolithiasis in patients with metabolic syndrome, focuses on modifying and reversing all these changes that these patients present, the treatment is composed of changes in the patient's lifestyle, and drugs that help in the modification of this factors that are altered in them.

Keywords: Metabolic syndrome, stones, cardiovascular risk, oxidative stress, uric acid, hypercalciuria.

* Médico pasante del servicio social en Investigación. Hospital
General de México, Unidad de Nefrología.

Facultad de Estudios Superiores Zaragoza (UNAM).

Recibido: 2 de Diciembre de 2019 Aceptado: 28 de Agosto de 2020.

\author{
Correspondencia: \\ Mariana Araceli Oseguera-Brizuela \\ Tel: 55 4818-8196 \\ E-mail: mariana200923@live.com.mx
}

Conflicto de Intereses: Todos los autores declaran que no existe ningún conflicto de intereses con respecto a la publicación de este artículo.

Citar como: Oseguera-Brizuela MA. Asociación de síndrome metabólico y nefrolitiasis.

El Residente. 2020; 15 (3): 89-96. https://dx.doi.org/10.35366/95959 


\section{INTRODUCCIÓN}

El síndrome metabólico es un conjunto de anormalidades metabólicas que incluyen obesidad central, hipertensión, dislipidemia e hiperglicemia o resistencia a la insulina. Todas estas entidades se correlacionan con la formación de litos, una patología relativamente frecuente en estos pacientes con una prevalencia de 8.8\% en Estados Unidos. Se deben principalmente a modificaciones en la orina debido a las alteraciones metabólicas que se desarrollan al padecer este síndrome.

La prevalencia del síndrome metabólico ha aumentado $30 \%$ en la última década, y aproximadamente $8.8 \%$ de estos pacientes llegan a padecer nefrolitiasis asociada con antecedente de síndrome metabólico, además de que la nefrolitiasis tiene un alto impacto económico en la población, ya que se estima que representa un gasto anual de 2,100 millones de dólares.

Cada uno de los componentes del síndrome metabólico se relaciona de alguna manera con la formación de litos renales, y éstos son importantes, ya sean aislados o en conjunto, para comprender la fisiopatología de la formación de éstos.

Uno de los componentes más importantes para comprender la fisiopatología y la asociación con el síndrome metabólico es el ácido úrico, el cual es una sustancia que aumenta en el organismo como consecuencia de una alteración en la homeostasis de estos pacientes, ya que el ácido úrico es uno de los principales componentes de los litos en estos pacientes así como el calcio, el cual sufre alteraciones similares, entre las que destacan alteraciones químicas en la orina de los pacientes que sufren síndrome metabólico.

Todos estos cambios en el metabolismo de estos pacientes traen consigo consecuencias que impactan en su vida, y aumentan el riesgo de muerte.

\section{DEFINICIÓN}

El síndrome metabólico es una combinación de factores sistémicos que incrementan el riesgo de enfermedad cardiovascular y diabetes melli- tus tipo 2. Puede definirse como un conjunto de anormalidades metabólicas que incluyen obesidad central, hipertensión arterial, dislipidemia, e hiperglicemia o resistencia a la insulina. ${ }^{1,2}$

Por otro lado, la nefrolitiasis puede definirse como la presencia de litos o piedras en riñón. Esta enfermedad es reconocida tradicionalmente como una patología aislada, benigna y de una condición realmente dolorosa. ${ }^{2}$

\section{COMPONENTES DEL SÍNDROME METABÓLICO}

La OMS (Organización Mundial de la Salud) establece que el síndrome metabólico se compone de:

- Presencia de glucosa anormal en ayuno, intolerancia a la glucosa, diabetes o resistencia a la insulina, más dos de los siguientes componentes:

- Circunferencia de cintura mayor de 90 $\mathrm{cm}$ en hombres, y mayor de $85 \mathrm{~cm}$ en mujeres.

- Nivel de triglicéridos mayor de $150 \mathrm{mg} /$ $\mathrm{dL}$ o c-HDL menor de 35 en hombres y menor de 39 en mujeres.

- Presión arterial mayor de 140/90 mmHg.

- Excreción urinaria de albúmina mayor de $20 \mu \mathrm{g} / \mathrm{min}$ o relación albumina-creatinina mayor de $30 \mu \mathrm{g}$.

Mientras que la ATP III (Adult treatment panel III) lo define como presencia de tres o más factores de riesgo:

- Obesidad abdominal (circunferencia de cintura) mayor de $102 \mathrm{~cm}$ en hombres $(90 \mathrm{~cm}$ en mexicanos), y mayor de $88 \mathrm{~cm}$ en mujeres (80 cm en mexicanas).

- Triglicéridos mayores o igual a $150 \mathrm{mg} / \mathrm{dL}$.

- c-HDL menor de $40 \mathrm{mg} / \mathrm{dL}$ en hombres y menor de $50 \mathrm{mg} / \mathrm{dL}$ en mujeres.

- Presión arterial mayor igual a 130/85 $\mathrm{mmHg}$ y glucosa en ayuno mayor igual a $100 \mathrm{mg} / \mathrm{dL} .^{3}$ 


\section{EPIDEMIOLOGÍA}

La prevalencia de cálculos renales se ha duplicado durante las tres décadas pasadas en Estados Unidos, de 2007 a 2010 los cálculos renales han afectado a $8.8 \%$ de la población de ese país. La población japonesa ha mostrado una ocurrencia anual de nefrolitiasis duplicada en un periodo de 30 años, al igual que la población estadounidense, mientras que en Reino Unido se estima que aproximadamente 720,000 individuos, sufren algún episodio de nefrolitiasis a lo largo de su vida. La proporción hombre-mujer es de $2: 1 .^{1-5}$

La nefrolitiasis además de ser una de las principales causas de morbilidad en el mundo, tiene un alto impacto económico; en el año 2000 el gasto anual total de nefrolitiasis fue estimado en 2,100 millones de dólares, representando un aumento de $50 \%$ de lo estimado en $1994 .^{6}$

En cuanto al riesgo de padecer nefrolitiasis en el paciente con síndrome metabólico, se ha demostrado que el riesgo que un paciente tiene de padecer nefrolitiasis incrementa conforme aumenta el número de componentes que presenta del síndrome metabólico, dando como resultado que los pacientes con tres o más componentes del síndrome tendrán mayor prevalencia de padecer nefrolitiasis. ${ }^{1}$

La litiasis renal es más común en lugares con alta humedad y temperatura elevada, por lo que se predice que con el aumento del calentamiento global, habrá un incremento de 1.6 a 2.2 millones de casos de litos renales a lo largo de la vida para el año 2050, particularmente en las regiones del sureste de Estados Unidos. ${ }^{7}$

En un estudio con ultrasonografía renal en Italia se encontró que la prevalencia de litos en pacientes con síndrome metabólico fue de $10.3 \%$, en pacientes hipertensos la prevalencia de litos renales fue de $13.3 \%$, contra $1.1 \%$ en pacientes normotensos, mientras que en otro estudio retrospectivo de cohorte transversal de Cirillo en 1988, la prevalencia de urolitiasis aumentó $1.1 \%$ en el grupo de personas con presión arterial baja $(<140 / 90 \mathrm{mmHg})$ contra $13.3 \%$ en sujetos con presión arterial elevada (175/115 mmHg o hipertensión tratada).
Por otro lado, en relación a diabetes mellitus en un estudio realizado en Turquía, se encontró que la prevalencia de nefrolitiasis fue mayor en pacientes diabéticos (21 vs. $8 \%$ de pacientes sanos). ${ }^{2,6}$

\section{COMPONENTES DEL SÍNDROME METABÓLICO Y SU ASOCIACIÓN CON LA FORMACIÓN DE LITOS}

Las razones de la asociación entre la formación litos y cada uno de los diversos procesos sistémicos que componen el síndrome metabólico no pueden señalarse de manera específica, pero pueden incluir responsabilidades metabólicas similares, hábitos dietéticos y mecanismos fisiopatológicos comunes. ${ }^{5}$

\section{Dislipidemia}

Se ha asociado significativamente con nefrolitiasis una elevación de los niveles de triglicéridos, siendo uno de los principales componentes del síndrome metabólico que propicia la formación de litos renales. ${ }^{1}$ Por otro lado, se ha relacionado aunque con menos significancia, con niveles séricos elevados de HDL y bajos niveles de lipoproteínas de baja densidad, los cuales influyen negativamente en la enfermedad cardiovascular y esto se asocia a su vez con un $\mathrm{pH}$ urinario disminuido en los pacientes. ${ }^{3}$

La dislipidemia se ha asociado con cambios que propician la formación de litos urinarios que incluyen hipercalciuria, hiperuricosuria, hiperoxaluria, y hipocitraturia; la elevación de triglicéridos y una reducción de HDL se han asociado con un incremento en el ácido úrico y por consiguiente, en la nefrolitiasis. ${ }^{4}$

\section{Obesidad}

La obesidad central se asocia con la ocurrencia y recurrencia de litos renales debido a las alteraciones metabólicas que éstos representan, se ha reportado una excreción de ácido úrico, sodio, calcio y citrato importantes en pacientes con obesidad. Esto se relaciona con la existencia 
de una acumulación de adipocitos tisulares, lo que incrementa la relación de adipocitos derivados de citosinas inflamatorias con otros factores potenciales del síndrome metabólico. ${ }^{1,7}$

La obesidad por sí sola no puede tener un rol clave en la formación de litos renales, pero tiene un efecto sistémico muy importante que involucra a todas las funciones del riñón, ocasionando diversos cambios en la orina que contribuyen a la formación de litos. ${ }^{2}$

\section{Hipertensión arterial}

La hipertensión arterial no ha mostrado un incremento relativamente importante en la incidencia de nefrolitiasis en los pacientes que la padecen, se ha observado que la nefrolitiasis y la hipertensión son desórdenes que siguen un espectro en común en la patogénesis de la enfermad como el estrés oxidativo, obesidad, enfermedad renal crónica, enfermedad coronaria, y ataque isquémico. Sin embargo y a pesar de que la compresión exacta de la relación entre ambas patologías permanece sombría, la asociación de hipertensión y de nefrolitiasis parece ser bidireccional. ${ }^{4,5}$

Se dice que la hipertensión tiene una asociación bidireccional con el riesgo de padecer nefrolitiasis porque los pacientes con hipertensión arterial han demostrado poseer cierto riesgo de desarrollar nefrolitiasis y los pacientes formadores de litos están predispuestos a desarrollar hipertensión arterial, comparados con pacientes sanos. El principal factor que puede contribuir a esta conexión epidemiológica y fisiopatológica es la hipercalciuria principalmente, aunque también se ha asociado con aterosclerosis y cambios microscópicos en el glomérulo y túbulo renal., ${ }^{4,6-8}$

Las perturbaciones del metabolismo de calcio se han vinculado posiblemente con el desarrollo de ambas, ya que se asocian las alteraciones de citrato urinario con el desarrollo de hipertensión arterial, y a su vez se dice que el citrato es influenciado por una alteración en el equilibrio ácido-base, por lo que es común encontrar hipocitraturia en pacientes con nefrolitiasis cálcica, lo que por otro lado se vincula con el desarrollo de hipertensión, mostrando esta asociación bidireccional existente entre ellas. ${ }^{2}$

\section{Diabetes}

Se ha reportado que pacientes que padecen diabetes tienen alta prevalencia de formar litos de ácido úrico así como los intolerantes a la glucosa. Esto se debe a que la resistencia a la insulina está asociada con una producción de amonio disminuida en el túbulo renal proximal, que ocasiona una disminución en el pH urinario, y éste es el principal factor asociado con la formación de litos de ácido úrico, ya que un $\mathrm{pH}$ urinario bajo es clave en la cristalización de ácido úrico. ${ }^{3,6,7}$

Al igual que en la hipertensión arterial, la diabetes y la nefrolitiasis tienen una relación bidireccional, por un lado la diabetes mellitus tipo 2 incrementa el riesgo de padecer litiasis, mientras que los pacientes que padecen nefrolitiasis tienen un incremento en el riesgo de padecer diabetes..$^{2,4}$

\section{FISIOPATOLOGÍA}

La fisiopatología de la formación de cálculos es un proceso complejo que incluye un volumen urinario bajo, hipercalciuria, hiperoxaluria, hipocitraturia, y anormalidades en el $\mathrm{pH}$ urinario. ${ }^{9}$

La formación de litos, principalmente de ácido úrico, es consecuencia de resistencia a la insulina, ya que si ésta disminuye la producción y transporte de amonio resultan en un $\mathrm{pH}$ urinario disminuido. Un aumento en la excreción de ácido úrico así como un pH disminuido son responsables de una sobresaturación urinaria. La excreción de oxalato se encuentra posiblemente relacionada con el IMC y la excreción de citrato depende de la dieta. ${ }^{1,10}$

Todos estos cambios producen cierto efecto en la cristalización de varias sales, lo que contribuye en la formación de litos renales. Esta cristalización es modulada por una gran variedad de macromoléculas, proteínas, carbohidratos y lípidos, algunos de éstos actúan como promotores de nucleación, crecimiento o agre- 
gación de cristales, mientras que otros actúan como inhibidores. ${ }^{7}$

\section{Ácido úrico}

Los pacientes formadores de litos de ácido úrico han demostrado una prevalencia significativamente alta de diabetes y una concentración elevada de triglicéridos séricos comparados con sujetos sanos. Se asocia la formación de piedras de ácido úrico con un aumento progresivo del IMC. ${ }^{2}$

El ácido úrico intracelular es postulado como una causa de resistencia a la insulina y gluconeogénesis aumentada; sin embargo, no se conocen exactamente las alteraciones del equilibrio extracelular e intracelular que sufren. Un incremento en el tejido adiposo puede aumentar la generación de ácido úrico, un exceso en fructuosa o sacarosa ingerida puede inducir obesidad junto a otros componentes del síndrome metabólico. ${ }^{8}$

La relación que existe entre hiperuricemia y elevación de triglicéridos no está realmente esclarecida, por otro lado un incremento en el ácido úrico sérico y una dieta alta en proteína animal puede causar una excreción elevada de ácido úrico por orina. La hiperuricosuria, especialmente en caso de bajo volumen urinario, puede resultar en la formación litos renales. ${ }^{8}$

\section{Hipercalciuria}

La hipercalcuria se define como la excreción urinaria de calcio en 24 horas mayor de $250 \mathrm{mg} /$ $\mathrm{dL}$ en mujeres y mayor de $300 \mathrm{mg} / \mathrm{dL}$ en hombres, entre $30-60 \%$ de los pacientes que presentan nefrolitiasis tienen hipercalciuria. ${ }^{9}$

Se debe a un defecto en la reabsorción de calcio en el túbulo renal con una elevación compensadora de PTH sérica y calcitriol, ocasionando un incremento en la absorción de calcio en el intestino o en la movilización a hueso. El síndrome metabólico influye en la excreción de calcio y en la supersaturación de oxalato de calcio. ${ }^{1}$

Con la hipercalciuria se relacionan varios componentes del síndrome metabólico como hipertensión arterial, dislipidemia, resistencia a la insulina, obesidad e igualmente se asocia con un IMC. ${ }^{10,11}$

\section{Anormalidades del $\mathrm{pH}$ urinario}

El pH urinario influye de manera muy significativa en la formación y composición de los litos, un $\mathrm{pH}$ urinario ácido promueve la formación de piedras de ácido úrico, mientras que un $\mathrm{pH}$ urinario alcalino propicia la formación de piedras de fosfato de calcio. ${ }^{1}$

$\mathrm{El} \mathrm{pH}$ urinario bajo reduce la solubilidad del ácido úrico generando la formación de los litos. Esto se atribuye principalmente a una generación inefectiva de amonio en el túbulo renal proximal, lo que se piensa está relacionado con resistencia a la insulina que promueve alteraciones hidroelectrolíticas en la excreción urinaria de 24 horas, lo que resulta en una orina más ácida y en un defecto en la excreción de ácido renal, además de hipocalciuria. ${ }^{3,6}$

Otro factor importante relacionado con la acidificación de la orina y la resistencia a la insulina es que se reduce directamente la generación tubular renal de amonio y se incrementa la reabsorción de sodio, produciendo orina más ácida como ya se comentó anteriormente. ${ }^{6}$

El mecanismo mediante el cual el síndrome metabólico puede disminuir el $\mathrm{pH}$ urinario incluye un incremento en el ácido titulable y una disminución de amonio, y citrato produciendo mayor concentración de iones hidrógeno y aumentando la orina ácida. ${ }^{4,5}$

Uno de los mecanismos que se ha visto implicado en la disminución del $\mathrm{pH}$ urinario y de amonio es que ambos disminuyen significativamente con un incremento en el número de componentes del síndrome metabólico. Como ya se mencionó anteriormente, experimentos en animales y en modelos de cultivo de células sugieren que estas respuestas pueden ser resultado de acumulación de lípidos dentro del riñón. ${ }^{5}$

\section{Estrés oxidativo}

El estrés oxidativo es un componente común en desórdenes cardiovasculares, incluyendo hi- 
pertensión, diabetes mellitus, aterosclerosis e infarto de miocardio, por lo que es sumamente importante en el desarrollo de nefrolitiasis. ${ }^{7}$

La obesidad y un exceso en ingesta de sal en pacientes con síndrome metabólico se asocian con una sobreproducción de especies reactivas de oxígeno y estrés oxidativo, lo que aumenta la evidencia de que las especies reactivas de oxígeno sólo son producidas durante la formación idiopática de piedras de oxalato de calcio renal. Se sugiere que la expresión de moléculas inflamatorias, y la infiltración de macrófagos activan las fases tempranas de formación de cálculos renales. ${ }^{9,10}$

La inflamación producida en este proceso fisiopatológico complejo generalmente se localiza alrededor de los depósitos de cristales en la papila renal, la exposición del epitelio renal a varios cristales se ha asociado con la producción de especies reactivas de oxígeno, la lesión en el epitelio promueve la formación y retención de cristales dentro de los riñones. ${ }^{7}$

Los factores proinflamatorios incrementan en pacientes obesos, mientras que los antiinflamatorios disminuyen; la concentración plasmática de un número de mediadores inflamatorios como FNT- $\alpha$, interleucina 6 , proteína $\mathrm{C}$ reactiva, inhibidores del factor de macrófagos migratorios se observa incrementada, mientras que la concentración de adiponectina se encuentra disminuida. ${ }^{7}$

\section{Hiperoxaluria}

Un factor altamente relacionado con este fenómeno es la dislipidemia, la cual por sí sola está asociada con hiperoxaluria (excreción urinaria de oxalato $\geq 45 \mathrm{mg} /$ día). También se relaciona con el sobrepeso, ya que el IMC es el mayor determinante de excreción urinaria de oxalato, y por otro lado la diabetes. Estos dos factores (sobrepeso y diabetes) han sido determinantes significativos en una excreción urinaria de oxalato elevado en adultos estadounidenses con y sin nefrolitiasis. ${ }^{10,11}$

La resistencia a la insulina, junto con un incremento en los ácidos grasos, disminuye la amoniogénesis renal, resultando en una disminución de la capacidad del búfer urinario, lo que también conlleva a una reducción del $\mathrm{pH}$ urinario e incrementa el riesgo de nefrolitiasis por ácido úrico y oxalato de calcio. La excreción de oxalato urinario ( $\geq 20 \mathrm{mg} /$ día) se asocia con un riesgo incrementado de nefrolitiasis por calcio. ${ }^{11,12}$

\section{PRINCIPAL COMPOSICIÓN DE LITOS EN PACIENTES CON SÍNDROME METABÓLICO Y NEFROLITIASIS}

Aproximadamente $75 \%$ de los cálculos urinarios son de calcio, de éstos, $80 \%$ son de oxalato de calcio, los cálculos de ácido úrico puro comprenden de 5 a $10 \%$ de los cálculos en estos pacientes, mientras que los cálculos relacionados con infecciones y estruvita forman parte de 10 a $15 \%$ de los cálculos; otros tipos de litos incluyen los relacionados con tratamientos con indinavir, xantina, triamtereno así como los de cistina que contribuyen a la formación de $<1 \% .^{9}$

En pacientes con obesidad los litos renales están compuestas generalmente de oxalato de calcio y ácido úrico, por lo que los pacientes con síndrome metabólico típicamente albergan piedras de estos compuestos. ${ }^{1,3}$

Las piedras de oxalato de calcio son las más prevalentes, seguidas de las de ácido úrico, mientras que las piedras de fosfato de calcio son las menos prevalentes en pacientes con síndrome metabólico. ${ }^{3}$

\section{COMPLICACIONES}

El síndrome metabólico se asocia con un aumento del riesgo de padecer enfermedades cardiovasculares, con enfermedades urológicas como disfunción eréctil, hiperplasia prostática benigna, enfermedad renal crónica y nefrolitiasis, además se asocia con hígado graso no alcohólico, síndrome de ovario poliquístico, apnea obstructiva del sueño, hipogonadismo, lipodistrofia, enfermedad microvascular y enfermedad renal crónica. ${ }^{1,4}$

Se ha encontrado que las enfermedades cardiovasculares así como la mortalidad son significativamente elevadas en pacientes formadores de litos renales con riesgo aumentado de padecer infarto agudo de miocardio, además de estar 
asociados con enfermedad arterial periférica y ateroesclerosis prematura. ${ }^{3,4}$

Por otro lado, se ha asociado un incremento en la mortalidad por nefrolitiasis relacionado con el desarrollo de cáncer en alguna parte del tracto urinario, incluso este aumento en la mortalidad es superior a la mortalidad por enfermedades cardiovasculares; sin embargo, el mecanismo implicado en el desarrollo de cáncer en estos pacientes aún no está claramente definido, pero se piensa que se debe a la irritación local e inflamación sistémica crónica y al lanzamiento incontable de citosinas inflamatorias que promueven la génesis del tumor. ${ }^{12}$

\section{Enfermedad cardiovascular}

La enfermedad cardiovascular es una de las principales consecuencias que traen consigo el síndrome metabólico y la nefrolitiasis, así como de las más peligrosas y con mayores índices de mortalidad. La enfermedad cardiovascular se vincula con inflamación crónica y estrés oxidativo, los cuales han sido propuestos como importantes factores que tienen un rol en la formación de litos renales. ${ }^{5}$

Los pacientes formadores de litos tienen mayor riesgo de padecer enfermedad coronaria arterial y de infarto agudo de miocardio, como ya se mencionó anteriormente. Un aumento en el espesor de la capa íntima y media de la arteria carótida ha mostrado estar directamente asociado con un incremento en el riesgo de padecer un infarto del miocardio. La aterosclerosis es un proceso progresivo, inicia años antes de que aparezcan las consecuencias clínicas, los adultos jóvenes que forman litos renales tienen una prevalencia más alta de aterosclerosis subclínica. El desarrollo de nefrolitiasis sintomática se ha asociado con un riesgo elevado de tener aterosclerosis de la arteria carótida. ${ }^{5,7}$

\section{TRATAMIENTO}

Se recomienda como parte del tratamiento no farmacológico reducir la ingesta de varias sustancias litogénicas como azúcares refinados, oxalato, purinas y sodio así como aumentar la ingesta de líquidos. Se recomienda dieta tipo DASH rica en vegetales y frutas, moderada en grasas, y baja en proteínas animales, lo cual es piedra angular en el tratamiento y prevención de esta patología, igualmente se recomienda realizar actividad física. ${ }^{1}$

El citrato de potasio actúa uniéndose al calcio ionizado, inhibiendo el oxalato de calcio, y la cristalización de fosfato de calcio, disminuyendo en orina el calcio ionizado e incrementando el citrato y $\mathrm{pH}$ urinario, por lo que puede emplearse.

Se pueden administrar diuréticos tiazídicos, principalmente hidroclorotiazida y clortalidona, ya que éstos actúan incrementando la reabsorción de calcio en el túbulo renal distal mediante la depleción de volumen extracelular en el túbulo renal proximal, y así disminuyen el calcio excretado en orina.

El alopurinol es otro fármaco que puede usarse en esta patología, ya que actúa inhibiendo la xantina oxidasa y mediante esta inhibición se logra disminuir tanto en suero como en orina el ácido úrico.

Otros fármacos que también pueden emplearse son el ácido acetohidroxámico, el cual inhibe la ureasa y disminuye pH así como la urea en orina, y el alfa-mercaptopropilglicerina que actúa mediante un intercambio de disulfuro disminuyendo la cistina excretada en orina. ${ }^{4}$

\section{PREVENCIÓN}

Se recomienda modificar el estilo de vida, en especial en pacientes obesos. Una dieta que incluya más frutas y vegetales, granos integrales, grasas monosaturadas, y productos bajos en grasas puede beneficiar a muchos pacientes con síndrome metabólico.

En la prevención de litos renales en pacientes con síndrome metabólico, se ha observado que la combinación de dieta y ejercicio tiene mayor efecto significativo que la dieta o el ejercicio aislados. ${ }^{1}$

Los componentes más importantes del síndrome metabólico, como se mencionó anteriormente, son: obesidad central, diabetes o resistencia a la insulina, e hipertensión arterial; 
estos componentes tienen en común que son causados por una ingesta alta de jarabe de fructuosa de maíz contenido en una gran variedad de bebidas y comida rápida. Esto es sumamente importante, ya que para prevenir el desarrollo de estas patologías, ya sea de manera aislada o en conjunto como síndrome, su consumo debe eliminarse o evitarse. ${ }^{7}$

\section{CONCLUSIONES}

El síndrome metabólico tiene una relación importante con el riesgo de padecer nefrolitiasis en estos pacientes, ya que fisiopatológicamente el síndrome metabólico produce cambios significativos en el organismo que propician la formación de litos como los cambios en el pH urinario, hipercalciuria, hiperoxaluria e hipocitraturia.

La nefrolitiasis incrementa el riesgo de padecer enfermedades cardiovasculares, y actualmente se encontró que también aumenta el riesgo de padecer cáncer del tracto urinario, lo que propicia un incremento en la mortalidad de estos pacientes.

Para disminuir el riesgo de padecer esta enfermedad es muy importante modificar el estilo de vida; principalmente la reducción del peso, ya que la obesidad es uno de los principales componentes del síndrome metabólico, y es uno de los factores que tiene más peso en los cambios que se producen en el organismo, junto con la intolerancia a la glucosa, para la formación de litos, por lo que una adecuada alimentación con una dieta rica en vegetales y frutas, granos integrales y baja en grasas, junto con el ejercicio, ayudará a disminuir el riesgo de formar litos, y de esta manera reducir las comorbilidades que esto conlleva.

Es importante conocer todos los cambios que el síndrome metabólico ocasiona en el organismo para así poder explicar a los pacientes cómo contribuyen en su estado de salud, así como las diferentes consecuencias que éstos pueden traer a su organismo. Todo ello con el fin de que ellos mismos lleven un control correcto de su enfermedad en conjunto con el equipo de salud, y que le den la importancia adecuada que ésta requiere. De esta forma se evitan las diferentes comorbilidades que traen estas patologías, se disminuye el riesgo de mortalidad, y se aumenta la esperanza y calidad de vida en estos pacientes.

\section{BIBLIOGRAFÍA}

1. Wong Y, Cook P, Roderick P, Bk S. Metabolic syndrome and kidney stone disease: a systematic review of literature. J Endourol. 2016; 30 (3): 246-253.

2. Sakhaee K. Nephrolithiasis as a systemic disorder. Curr Opin Nephrol Hypertens. 2008; 17 (3): 304-309.

3. Boyd C, Wood K, Whitaker D, Assimos DG. The influence of metabolic syndrome and its components on the development of nephrolithiasis. Asian J Urol. 2018; 5 (4): 215-222. Available from: https://doi.org/10.1016/j. ajur.2018.06.002.

4. Dibianco JM, Jarrett TW, Mufarrij P. Metabolic syndrome and nephrolithiasis risk: should the medical management of nephrolithiasis include the treatment of metabolic syndrome? Rev Urol. 2015; 17 (3): 117-128.

5. Lange JN, Mufarrij PW, Wood KD, Holmes RP, Assimos DG. The association of cardiovascular disease and metabolic syndrome with nephrolithiasis. Curr Opin Urol. 2012; 22 (2): 154-159.

6. Spatola L, Angelini C, Badalamenti S, Maringhini S, Gambaro G. Kidney stones diseases and glycaemic statuses: focus on the latest clinical evidences. Urolithiasis. 2017; 45 (5): 457-460.

7. Khan SR. Is oxidative stress, a link between nephrolithiasis and obesity, hypertension, diabetes, chronic kidney disease, metabolic syndrome? Urol Res. 2012; 40 (95): 95-112.

8. Sharaf UA, Din E, Salem MM, Abdulazim DO. Uric acid in the pathogenesis of metabolic, renal, and cardiovascular diseases: a review. J Adv Res. 2017; 8 (5): 537-548. Available from: http://dx.doi.org/10.1016/j. jare.2016.11.004.

9. Shadman A, Bastani B. Kidney Calculi: Pathophysiology and as a systemic disorder. Iran J Kidney Dis. 2017; 11 (3): 180-191.

10. Carbone A, Salhi YA, Tasca A, Palleschi G, Fuschi A, Nunzio CD et al. Obesity and kidney stone disease: a systematic review. Minerva Uologica Nefrol. 2018; 70 (4): 393-400.

11. Ticinesi A, Guerra A, Allegri F, Nouvenne A. Determinants of calcium and oxalate excretion in subjects with calcium nephrolithiasis: the role of metabolic syndrome traits. J Nephrol. 2018; 31: 395-403. Available from: http://dx.doi.org/10.1007/s40620-017-0453-3.

12. Dhondup T, Kittanamongkolchai W, Vaughan LE, Mehta RA, Chhina JK, Enders FT et al. Risk of ESRD and mortality in kidney and bladder stone formers. Am J Kidney Dis. 2018; 72 (6): 790-797. Available from: https:// doi.org/10.1053/j.ajkd.2018.06.012. 